\title{
GENERALIZED SHARP HARDY TYPE AND CAFFARELLI-KOHN-NIRENBERG TYPE INEQUALITIES ON RIEMANNIAN MANIFOLDS
}

\author{
SHIHSHU WALTER WEI* AND YE LI
}

\begin{abstract}
We prove generalized Hardy's type inequalities with sharp constants and Caffarelli-Kohn-Nirenberg inequalities with sharp constants on Riemannian manifolds $M$. When the manifold is Euclidean space we recapture the sharp CaffarelliKohn-Nirenberg inequality. By using a double limiting argument, we obtain an inequality that implies a sharp Hardy's inequality, for functions with compact support on the manifold $M$ (that is, not necessarily on a punctured manifold $M \backslash\left\{x_{0}\right\}$ where $x_{0}$ is a fixed point in $M$ ). Some topological and geometric applications are discussed.
\end{abstract}

\section{Introduction}

It is well-known that Hardy-type inequalities have been widely used in analysis and differential equations. In [5] Caffarelli, Kohn and Nirenberg proved rather general interpolation inequalities with weights. C.S. Lin [14] has generalized these results to include derivatives of all orders.

On the other hand, completing the square, in particular the elementary result that $a t^{2}+b t+c \geq 0$ with $a>0$ holds for all $t \in \mathbb{R}$, if and only if $b^{2}-4 a c \leq 0$, has far-reaching consequences or analogs in various branches of mathematics. These include the Cauchy-Schwarz inequality in Hilbert space, the Bochner method in differential geometry, the $L^{2}$ method in several complex variables, the Kodaira vanishing theorem and Kodaira imbedding theorem in Kähler geometry, geometric inequalities in partial differential equations, and curvature estimates in submanifold theory (see e.g. [2], [11], [8], [13], [12]).

Corresponding author: S. W. Wei .

2000 Mathematics Subject Classification. Primary: 26D15, 53C21; Secondary 53C20.

Key words and phrases. Caffarelli-Kohn-Nirenberg inequality, Hardy inequality. radial curvature, Hessian comparison theorem, sectional curvature.

${ }^{*}$ Research was partially supported by NSF Award No DMS-0508661, OU Presidential International Travel Fellowship, and OU Faculty Enrichment Grant. 
Using only integration by parts and the elementary algebraic inequality mentioned above, D. G. Costa provides a new and short proof for the $L^{2}$ version of the CaffarelliKohn-Nirenberg inequality with sharp constants:

$$
\breve{C} \int_{\mathbb{R}^{n}} \frac{|u|^{2}}{|x|^{(a+b+1)}} d x \leq\left(\int_{\mathbb{R}^{n}} \frac{|u|^{2}}{|x|^{2 a}} d x\right)^{\frac{1}{2}}\left(\int_{\mathbb{R}^{n}} \frac{|\nabla u|^{2}}{|x|^{2 b}} d x\right)^{\frac{1}{2}}
$$

for $u \in C_{0}^{\infty}\left(\mathbb{R}^{n} \backslash\{0\}\right)$, where $a, b \in \mathbb{R}$ and $\breve{C}=\breve{C}(a, b)=\frac{|n-(a+b+1)|}{2}$ is sharp.

When $a=1$ and $b=0$, one recaptures Hardy's inequality

$$
\left(\frac{n-2}{2}\right)^{2} \int_{\mathbb{R}^{n}} \frac{|u|^{2}}{|x|^{2}} d v \leq \int_{\mathbb{R}^{n}}|\nabla u|^{2} d v
$$

for $u \in C_{0}^{\infty}\left(\mathbb{R}^{n} \backslash\{0\}\right)$, where the constant $\left(\frac{n-2}{2}\right)^{2}$ is sharp (cf. [6]).

In the first part of this article, by using a double limiting argument, we obtain an inequality that implies a sharp Hardy's inequality, for functions with compact support on the manifold $M$ (that is, not necessarily on a punctured manifold $M \backslash\left\{x_{0}\right\}$ where $x_{0}$ is a fixed point in $M$ ). Since for general manifolds $M$, the length $|x|$, of a point in $M$ is not defined for $x \in M$, the Caffarelli-Kohn-Nirenberg inequality (1.1) and Hardy's inequality (1.2) do not seem to carry over immediately to manifolds. The techniques we use are the Hessian comparison theorem by constructing appropriate vector fields that involve a radial vector field on $M$. Throughout the whole paper, unless it is stated otherwise, we let $M$ be an $n$-dimensional Riemannian manifold with a pole $x_{0}, r$ be the distance function defined on $M$ from the pole $x_{0} \in M, \Delta r$ be the Laplacian of $r$ (cf. [7] or Section $2)$, and $1<p<\infty$.

Theorem 1. For every $u \in W_{0}^{1, p}(M)$ and every $\epsilon>0$, the following inequality holds:

$$
\begin{aligned}
& \left.\left|-\int_{\partial B_{\delta}\left(x_{0}\right)} \frac{r}{r^{p}+\epsilon}\right| u\right|^{p} d S+\int_{M \backslash B_{\delta}\left(x_{0}\right)} \frac{\left(r^{p}+\epsilon\right)(r \Delta r+1)-p r^{p}}{\left(r^{p}+\epsilon\right)^{2}}|u|^{p} d v \mid \\
& \quad \leq p\left(\int_{M \backslash B_{\delta}\left(x_{0}\right)}\left(\frac{|u|^{p-1} r}{r^{p}+\epsilon}\right)^{\frac{p}{p-1}} d v\right)^{\frac{p-1}{p}}\left(\int_{M \backslash B_{\delta}\left(x_{0}\right)}|\nabla u|^{p} d v\right)^{\frac{1}{p}}
\end{aligned}
$$

for sufficiently small $\delta>0$, where $\partial B_{\delta}\left(x_{0}\right)$ denotes the $C^{1}$ boundary of the geodesic ball $B_{\delta}\left(x_{0}\right)$ centered at $x_{0}$ with radius $\delta>0, d S$ and $d v$ are the volume element of $\partial B_{\delta}\left(x_{0}\right)$ and $M$ respectively. In particular, if $M$ is manifold of radial curvature $K \leq 0$ with $n>p$. Then $\frac{|u|^{p}}{r^{p}} \in L^{1}(M)$, and

$$
\left(\frac{n-p}{p}\right)^{p} \int_{M} \frac{|u|^{p}}{r^{p}} d v \leq \int_{M}|\nabla u|^{p} d v
$$

for every $u \in W_{0}^{1, p}(M)$. 
This result is sharp when $M=\mathbb{R}^{n}$.

Corollary 1.1. Let $M$ be a manifold of radial curvature $K \leq 0$ with $n>2$. Then for every $u \in W_{0}^{1,2}(M)$,

$$
\left(\frac{n-2}{2}\right)^{2} \int_{M} \frac{|u|^{2}}{r^{2}} d v \leq \int_{M}|\nabla u|^{2} d v ;
$$

This result is sharp when $M=\mathbb{R}^{n}$. Moreover, this theorem does not require $u \in$ $W_{0}^{1,2}\left(M \backslash\left\{x_{0}\right\}\right)$ and hence is stronger than the result obtained from inequality (1.9) in Theorem 3 by setting $a=1$ and $b=0$.

Corollary 1.2. Let $M$ be a complete simply connected manifold of sectional curvature $\kappa \leq 0$. Then (1.4) holds for every $x_{0} \in M$, for every $u \in W_{0}^{1, p}(M)$, with $p<n$.

Theorem 2. Let $M$ be a manifold of radial curvature $K \geq 0$ with $n<p$. Then for every $u \in W_{0}^{1, p}(M), \frac{|u|^{p}}{r^{p}} \in L^{1}(M)$, and the following inequality holds:

$$
\left(-\frac{n-p}{p}\right)^{p} \int_{M} \frac{|u|^{p}}{r^{p}} d v \leq \int_{M}|\nabla u|^{p} d v
$$

As an immediate consequence, one has

Corollary 1.3. Let $M$ be an elliptic n-paraboloid in $\mathbb{R}^{n+1}$. Then the conclusion of Theorem 2 holds, where $x_{0}$ is the vertex of $M$.

Corollary 1.4. Let $K:[0, \infty) \rightarrow R$ be a smooth function satisfying $K \geq 0$ and $\int_{0}^{\infty} s K(s) d s \leq 1$, and $f$ be a smooth solution of the differential equation $f^{\prime \prime}=-K f$, with initial conditions $f(0)=0$ and $f^{\prime}(0)=1$. Let $M=\mathbb{R}^{n}$ with a smooth metric $\tilde{g}$ such that $\tilde{g}$ restricted to $\mathbb{R}^{n} \backslash\{0\}$ is $g=d r^{2}+f(r)^{2} d \Theta$, where $r$ is the radial function on $\mathbb{R}^{n}$ and $d \Theta$ is the tensor which restricts to the usual metric on sphere around the origin 0 . Then (1.6) holds for every $u \in W_{0}^{1, p}(M)$, with $p>n$.

Corollary 1.5. Let $M$ be a manifold of radial curvature $K \equiv 0$. Then for every $u \in$ $W_{0}^{1, p}(M)$, the following inequality holds:

$$
\left|\frac{n-p}{p}\right|^{p} \int_{M} \frac{|u|^{p}}{r^{p}} d v \leq \int_{M}|\nabla u|^{p} d v
$$

Corollary 1.5 is sharp when the smooth function $K \equiv 0$ and recaptures the $L^{p}$ version of the Hardy's inequality in $\mathbb{R}^{n}$ (see e.g. [15]).

In the second part of this article, we generalize the above inequality (1.1) in Euclidean space to a class of Caffarelli-Kohn-Nirenberg type inequalities with sharp constants on Riemannian manifolds. We use the Hessian comparison theorem and Costa's approach of employing the elementary algebraic inequality $b^{2}-4 a c \leq 0$, by constructing appropriate vector fields that involve a radial vector field on $M$. 
Theorem 3. For every $u \in W_{0}^{1,2}\left(M \backslash\left\{x_{0}\right\}\right)$, and every $a, b \in \mathbb{R}$, the following inequality holds:

$$
\begin{aligned}
& \frac{1}{2}\left|\int_{M} \frac{|u|^{2}}{r^{a+b+1}}(r \Delta r-a-b) d v\right| \\
& \quad \leq\left(\int_{M} \frac{|u|^{2}}{r^{2 a}} d v\right)^{\frac{1}{2}}\left(\int_{M} \frac{|\nabla u|^{2}}{r^{2 b}} d v\right)^{\frac{1}{2}}
\end{aligned}
$$

where $d v$ is the volume element of $M$. In particular, if $M$ is a manifold of radial curvature $K \leq 0$. then for every $u \in W_{0}^{1,2}\left(M \backslash\left\{x_{0}\right\}\right)$, and every $a, b \in \mathbb{R}$ with $a+b+1 \leq n$,

$$
\tilde{C} \int_{M} \frac{|u|^{2}}{r^{a+b+1}} d v \leq\left(\int_{M} \frac{|u|^{2}}{r^{2 a}} d v\right)^{\frac{1}{2}}\left(\int_{M} \frac{|\nabla u|^{2}}{r^{2 b}} d v\right)^{\frac{1}{2}}
$$

where $d v$ is the volume element of $M$ and $\tilde{C}=\tilde{C}(a, b):=\frac{n-(a+b+1)}{2}$ is a constant.

Corollary 1.6. Let $M$ be a complete simply connected manifold of sectional curvature $\kappa \leq 0$. Then (1.9) holds for every $x_{0} \in M$, for every $u \in W_{0}^{1,2}\left(M \backslash\left\{x_{0}\right\}\right)$, and every $a, b \in \mathbb{R}$ with $a+b+1 \leq n$.

This corollary is sharp when the sectional curvature $\kappa \equiv 0$ and recaptures the $L^{2}$ version of Caffarelli-Kohn-Nirenberg inequality in $\mathbb{R}^{n}$ by Cartan-Ambrose-Hicks Theorem (cf. $[1,4,9,10])$.

Theorem 4. Let $M$ be a manifold of radial curvature $K \geq 0$. Then for every $u \in$ $W_{0}^{1,2}\left(M \backslash\left\{x_{0}\right\}\right)$, and every $a, b \in \mathbb{R}$ with $a+b+1 \geq n$, the following inequality holds:

$$
\hat{C} \int_{M} \frac{|u|^{2}}{r^{a+b+1}} d v \leq\left(\int_{M} \frac{|u|^{2}}{r^{2 a}} d v\right)^{\frac{1}{2}}\left(\int_{M} \frac{|\nabla u|^{2}}{r^{2 b}} d v\right)^{\frac{1}{2}}
$$

where $\hat{C}=\hat{C}(a, b):=-\frac{n-(a+b+1)}{2}$ is a constant.

As an immediate consequence, one has

Corollary 1.7. Let $M$ be an elliptic $n$-paraboloid in $\mathbb{R}^{n+1}$. Then the conclusion of Theorem 4 holds, where $x_{0}$ is the vertex of $M$.

Corollary 1.8. Let $K, f, M$ and $r$ be as in Corollary 1.4. Then (1.10) holds for every $u \in W_{0}^{1,2}(M \backslash\{0\})$, and every $a, b \in \mathbb{R}$ with $a+b+1 \geq n$.

Corollary 1.8 is sharp when the smooth function $K \equiv 0$ and recaptures the $L^{2}$ version of the Caffarelli-Kohn-Nirenberg inequality in $\mathbb{R}^{n}$ by Proposition 4.2 in [7]. 
Corollary 1.9. Let $M$ be a manifold of radial curvature $K \equiv 0$. Then for every $u \in$ $W_{0}^{1,2}\left(M \backslash\left\{x_{0}\right\}\right)$, and every $a, b \in \mathbb{R}$, then the following inequality holds:

$$
\check{C} \int_{M} \frac{|u|^{2}}{r^{a+b+1}} d v \leq\left(\int_{M} \frac{|u|^{2}}{r^{2 a}} d v\right)^{\frac{1}{2}}\left(\int_{M} \frac{|\nabla u|^{2}}{r^{2 b}} d v\right)^{\frac{1}{2}}
$$

where $\check{C}=\check{C}(a, b):=\left|\frac{n-(a+b+1)}{2}\right|$ is a constant.

In [17], knowledge of an essential positive supersolution of a nonlinear P.D.E. yields information on topology and geometry. Analogously, in the third part of this article, we will discuss some topological and geometric applications of these inequalities (cf. Theorems 7 and 8).

\section{Manifolds which possess a pole and The Hessian Comparison Theorem}

We recall some related basic facts, notations, definitions, and formulas (see [7] for details).

\subsection{Manifolds which possess a pole}

A Cartan-Hadamard manifold is a complete simply-connected Riemannian manifold of nonpositive sectional curvature. We denote $T_{x_{0}} M$ the tangent space to $M$ at $x_{0} \in M$. A pole is a point $x_{0} \in M$ such that the exponential map $\exp _{x_{0}}: T_{x_{0}} M \rightarrow M$ is a diffeomorphism. The theorem of Cartan-Hadamard states that if $M$ is a Cartan-Hadamard manifold, and $x \in M$, then the exponential map $\exp _{x}: T_{x} M \rightarrow M$ is a diffeomorphism. Thus every point of a Cartan-Hadamard manifold is a pole. Furthermore, if $M$ possess a pole, $M$ is complete. Given such a manifold $M$ with a pole $x_{0}$, the radial vector field $\partial$ on $M \backslash\left\{x_{0}\right\}$ is the unit vector field such that for any $x \in M \backslash\left\{x_{0}\right\}, \partial(x)$ is the unit vector tangent to the unique geodesic joining $x_{0}$ to $x$ and pointing away from $x_{0}$. A radial plane is a plane $\pi$ which contains $\partial(x)$ in the tangent space $T_{x} M$. By the radial curvature $K$ of a manifold with a pole, we mean the restriction of the sectional curvature function to all the radial planes. We define $K(t)$ to be the radial curvature of $M$ at $x$ for any $x$ such that $r(x)=t$.

\subsection{The Hessian Comparison Theorem}

Let $(M, g)$ be a manifold with a pole $x_{0}$. Let a tensor $g-d r \bigotimes d r=0$ on the radial direction, and is just the metric tensor $g$ on the orthogonal complement $\partial^{\perp}$. We recall the following:

Theorem 5. (cf. [7]) (i) If $-\alpha^{2} \leq K(r) \leq-\beta^{2}$ with $\alpha>0, \beta>0$, then

$$
\beta \operatorname{coth}(\beta r)[g-d r \otimes d r] \leq H e s s(r) \leq \alpha \operatorname{coth}(\alpha r)[g-d r \otimes d r]
$$


(ii) If $-\frac{a}{1+r^{2}} \leq K(r) \leq 0$ with $a \geq 0$, then

$$
\frac{1}{r}[g-d r \otimes d r] \leq \operatorname{Hess}(r) \leq \frac{1+\sqrt{1+4 a}}{2 r}[g-d r \otimes d r]
$$

(iii) If $0 \leq K(r) \leq \frac{b}{1+r^{2}}$ with $b \in[0,1 / 4]$, then

$$
\frac{1+\sqrt{1-4 b}}{2 r}[g-d r \otimes d r] \leq H e s s(r) \leq \frac{1}{r}[g-d r \otimes d r]
$$

(iv) If $-A r^{2 q} \leq K(r) \leq-B r^{2 q}$ with $A \geq B>0$ and $q>0$, then

$$
B_{0} r^{q}[g-d r \otimes d r] \leq H e s s(r) \leq(\sqrt{A} \operatorname{coth} \sqrt{A}) r^{q}[g-d r \otimes d r]
$$

for $r \geq 1$, where $B_{0}=\min \left\{1,-\frac{q+1}{2}+\left[B+\left(\frac{q+1}{2}\right)^{2}\right]^{1 / 2}\right\}$.

\section{A generalized Hardy's inequality with sharp constants on manifolds}

In this section we use a double limiting argument to prove a generalized Hardy's inequality with sharp constants on manifolds.

Proof of Theorem 1. For any given $u \in W_{0}^{1, p}(M)$, and any given $\epsilon>0$, we let $V$ be an open set with $C^{1}$ boundary $\partial V$, such that $V \subset \subset M$, and $u=0$ off $V$. We first choose a sufficiently small $\delta>0$, so that $\partial V \cap \partial B_{\delta}\left(x_{0}\right)=\emptyset$. We then consider $I:=p \int_{M \backslash B_{\delta}\left(x_{0}\right)}\left\langle|u|^{p-2} u \frac{r \partial}{r^{p}+\epsilon}, \nabla u\right\rangle d v$. It follows from the Guass lemma that

$$
I=\int_{M \backslash B_{\delta}\left(x_{0}\right)} \operatorname{div}\left(\frac{r \partial}{r^{p}+\epsilon}|u|^{p}\right) d v-\int_{M \backslash B_{\delta}\left(x_{0}\right)} \frac{\operatorname{div}(r \partial)}{r^{p}+\epsilon}|u|^{p} d v+\int_{M \backslash B_{\delta}\left(x_{0}\right)} \frac{p r^{p}}{\left(r^{p}+\epsilon\right)^{2}}|u|^{p} d v
$$

By the divergence theorem, and the fact that the unit outward normal vector $\nu$ on $\partial B_{\delta}\left(x_{0}\right)$ is $-\partial$, the first term in the right hand side of (3.1) satisfies

$$
\begin{aligned}
\int_{M \backslash B_{\delta}\left(x_{0}\right)} \operatorname{div}\left(\frac{r \partial}{r^{p}+\epsilon}|u|^{p}\right) d v & =\int_{V \backslash B_{\delta}\left(x_{0}\right)} \operatorname{div}\left(\frac{r \partial}{r^{p}+\epsilon}|u|^{p}\right) d v \\
& =-\int_{\partial B_{\delta}\left(x_{0}\right)}\left\langle\frac{r \partial}{r^{p}+\epsilon}|u|^{p}, \nu\right\rangle d S \\
& =\int_{\partial B_{\delta}\left(x_{0}\right)} \frac{r}{r^{p}+\epsilon}|u|^{p} d S
\end{aligned}
$$

Let $\left\{e_{i}\right\}_{i=1}^{n}$ be a local orthonormal frame field on $M$ such that $e_{1}=\partial$. Denote $\nabla$ the Riemannian connection on $M$. Then $\nabla_{\partial} \partial=0$ in $M$ and the Hessian of $r$ is given by 
$\left(\nabla_{e_{i}} d r\right)\left(e_{i}\right)=\nabla_{e_{i}}\left(d r\left(e_{i}\right)\right)-d r\left(\nabla_{e_{i}} e_{i}\right)$. Furthermore, off $B_{\delta}\left(x_{0}\right)$

$$
\begin{aligned}
\operatorname{div}(\partial) & =\left\langle\nabla_{\partial} \partial, \partial\right\rangle+\sum_{i=2}^{n}\left\langle\nabla_{e_{i}}(\partial), e_{i}\right\rangle \\
& =\sum_{i=2}^{n}\left(\nabla_{e_{i}} d r\right)\left(e_{i}\right) \\
& =\sum_{i=2}^{n} \operatorname{Hess}(r)\left(e_{i}, e_{i}\right)
\end{aligned}
$$

where $\operatorname{Hess}(r)$ is the Hessian of $r$. By the Gauss lemma, $\nabla r=\partial$,

$$
\nabla\left(r^{p}+\epsilon\right)^{-1}=-\left(r^{p}+\epsilon\right)^{-2} p r^{p-1} \partial
$$

Substituting (3.2)-(3.4) into (3.1), one has

$$
-\int_{\partial B_{\delta}\left(x_{0}\right)} \frac{r}{r^{p}+\epsilon}|u|^{p} d S+\int_{M \backslash B_{\delta}\left(x_{0}\right)} \frac{\left(r^{p}+\epsilon\right)\left[\sum_{i=2}^{n} r \operatorname{Hess}(r)\left(e_{i}, e_{i}\right)+1\right]-p r^{p}}{\left(r^{p}+\epsilon\right)^{2}}|u|^{p} d v=-I
$$

In view of Hölder inequality, and the fact $\sum_{i=2}^{n} r \operatorname{Hess}(r)\left(e_{i}, e_{i}\right)+1=r \Delta r+1$, one obtains the desired (1.3).

When $K \leq 0$, by assumption and the Hessian comparison theorem, $r \Delta r+1 \geq n>p$. It follows from (1.3) that

$$
\begin{aligned}
& -\int_{\partial B_{\delta}\left(x_{0}\right)} \frac{r}{r^{p}+\epsilon}|u|^{p} d S+\int_{M \backslash B_{\delta}\left(x_{0}\right)} \frac{(n-p) r^{p}+n \epsilon}{\left.r^{p}+\epsilon\right)^{2}}|u|^{p} d v \\
& \quad \leq p\left(\int_{M \backslash B_{\delta}\left(x_{0}\right)} \frac{\left(r^{p}\right)^{\frac{1}{p-1}}}{\left(r^{p}+\epsilon\right)^{\frac{p}{p-1}}}|u|^{p} d v\right)^{\frac{p-1}{p}}\left(\int_{M \backslash B_{\delta}\left(x_{0}\right)}|\nabla u|^{p} d v\right)^{\frac{1}{p}}
\end{aligned}
$$

Furthermore,

$$
\begin{aligned}
\int_{M \backslash B_{\delta}\left(x_{0}\right)} \frac{(n-p) r^{p}+n \epsilon}{\left(r^{p}+\epsilon\right)^{2}}|u|^{p} d v & \geq \int_{M \backslash B_{\delta}\left(x_{0}\right)} \frac{(n-p) r^{p}+n \epsilon-p \epsilon}{\left(r^{p}+\epsilon\right)^{2}}|u|^{p} d v \\
& \geq(n-p) \int_{M \backslash B_{\delta}\left(x_{0}\right)} \frac{\left(r^{p}+\epsilon\right)^{\frac{1}{p-1}}}{\left(r^{p}+\epsilon\right)^{\frac{p}{p-1}}}|u|^{p} d v \\
& \geq(n-p) \int_{M \backslash B_{\delta}\left(x_{0}\right)} \frac{\left(r^{p}\right)^{\frac{1}{p-1}}}{\left(r^{p}+\epsilon\right)^{\frac{p}{p-1}}}|u|^{p} d v
\end{aligned}
$$

Substituting (3.7) into (3.6), one gets

$$
\begin{aligned}
& -\int_{\partial B_{\delta}\left(x_{0}\right)} \frac{r}{r^{p}+\epsilon}|u|^{p} d S+(n-p) \int_{M \backslash B_{\delta}\left(x_{0}\right)} \frac{\left(r^{p}\right)^{\frac{1}{p-1}}}{\left(r^{p}+\epsilon\right)^{\frac{p}{p-1}}}|u|^{p} d v \\
& \quad \leq p\left(\int_{M \backslash B_{\delta}\left(x_{0}\right)} \frac{\left(r^{p}\right)^{\frac{1}{p-1}}}{\left(r^{p}+\epsilon\right)^{\frac{p}{p-1}}}|u|^{p} d v\right)^{\frac{p-1}{p}}\left(\int_{M \backslash B_{\delta}\left(x_{0}\right)}|\nabla u|^{p} d v\right)^{\frac{1}{p}}
\end{aligned}
$$


Thus, by taking sufficiently small $\delta>0$, one has

$$
\int_{\partial B_{\delta}\left(x_{0}\right)} \frac{r}{r^{p}+\epsilon}|u|^{p} d S=0, \quad \text { if } \quad x_{0} \notin V
$$

and

$$
0 \leq \int_{\partial B_{\delta}\left(x_{0}\right)} \frac{r}{r^{p}+\epsilon}|u|^{p} d S \leq \frac{\delta}{\delta^{p}+\epsilon}|u|_{L^{p}(M)}^{p} \rightarrow 0 \quad \text { as } \delta \rightarrow 0, \quad \text { if } \quad x_{0} \in V .
$$

As $\delta \rightarrow 0$, (3.8), via (3.9) or (3.10) implies that for every $\epsilon>0$,

$$
(n-p) \int_{M} \frac{\left(r^{p}\right)^{\frac{1}{p-1}}}{\left(r^{p}+\epsilon\right)^{\frac{p}{p-1}}}|u|^{p} d v \leq p\left(\int_{M} \frac{\left(r^{p}\right)^{\frac{1}{p-1}}}{\left(r^{p}+\epsilon\right)^{\frac{p}{p-1}}}|u|^{p} d v\right)^{\frac{p-1}{p}}\left(\int_{M}|\nabla u|^{p} d v\right)^{\frac{1}{p}}
$$

Letting $\epsilon \rightarrow 0$ gives the desired (1.4).

Proof of Theorem 2. When $K \geq 0$, by assumption and the Hessian comparison theorem, $r \Delta r+1 \leq n<p$. By letting $\delta \rightarrow 0$ in (1.3), one has

$$
\int_{M} \frac{(p-n) r^{p}-n \epsilon}{\left(r^{p}+\epsilon\right)^{2}}|u|^{p} d v \leq p\left(\int_{M}\left(\frac{|u|^{p-1} r}{r^{p}+\epsilon}\right)^{\frac{p}{p-1}} d v\right)^{\frac{p-1}{p}}\left(\int_{M}|\nabla u|^{p} d v\right)^{\frac{1}{p}}
$$

We observe that the factor $\frac{r}{r^{p}+\epsilon}$ on the right hand side of (3.12) is uniformly bounded on $M$. By the dominate convergent theorem, we let $\epsilon$ tends to 0 and obtain the desired (1.6).

Proof of Corollary 1.4. It follows from the construction in the proof of Proposition 4.2 in [7] that $M$ is a manifold with a pole, and the preassigned function $K$ is the radial curvature of $M$. Now the result follows from Theorem 2 .

\section{Proof of Theorems 3 and 4}

For every $u \in W_{0}^{1,2}\left(M \backslash\left\{x_{0}\right\}\right)$ and every $a, b, t \in \mathbb{R}$, we have

$$
\int_{M}\left|\frac{\nabla u}{|r \partial|^{b}}+t \frac{\partial}{r^{a}|\partial|^{a+1}} u\right|^{2} d v \geq 0
$$

where $\nabla u$ is the weak derivative of $u$. Since $|\partial|=1,(4.1)$ becomes

$$
\int_{M} \frac{|\nabla u|^{2}}{r^{2 b}} d v+t^{2} \int_{M} \frac{|u|^{2}}{r^{2 a}} d v+2 t \int_{M}\left\langle u \frac{\partial}{r^{a+b}}, \nabla u\right\rangle d v \geq 0
$$

Denote the last integral by $I I$. In view of the product rule,

$$
I I=\int_{M} \operatorname{div}\left(u \frac{\partial}{r^{a+b}} u\right) d v-I I-\int_{M} \frac{|u|^{2}}{r^{a+b}} \operatorname{div}(\partial) d v-\int_{M}|u|^{2}\left\langle\partial, \nabla r^{-(a+b)}\right\rangle d v
$$


By the divergence theorem, the first term in the right hand side of (4.3) satisfies

$$
\int_{M} \operatorname{div}\left(u \frac{\partial}{r^{a+b}} u\right) d v=\int_{V} \operatorname{div}\left(u \frac{\partial}{r^{a+b}} u\right) d v=0
$$

where $V$ is an open set with $C^{1}$ boundary and $\operatorname{supp}\{u\} \subset V \subset \subset M \backslash\left\{x_{0}\right\}$. Let $\left\{e_{i}\right\}_{i=1}^{n}$ be a local orthonormal frame field on $M \backslash\left\{x_{0}\right\}$ such that $e_{1}=\partial$. Denote $\nabla$ the Riemannian connection on $M \backslash\left\{x_{0}\right\}$. Then $\nabla_{\partial} \partial=0$ in $M \backslash\left\{x_{0}\right\}$ and the Hessian of $r$ is given by $\left(\nabla_{e_{i}} d r\right)\left(e_{i}\right)=\nabla_{e_{i}}\left(d r\left(e_{i}\right)\right)-d r\left(\nabla_{e_{i}} e_{i}\right)$. Furthermore,

$$
\begin{aligned}
\operatorname{div}(\partial) & =\left\langle\nabla_{\partial} \partial, \partial\right\rangle+\sum_{i=2}^{n}\left\langle\nabla_{e_{i}}(\partial), e_{i}\right\rangle \\
& =\sum_{i=2}^{n}\left(\nabla_{e_{i}} d r\right)\left(e_{i}\right) \\
& =\sum_{i=2}^{n} \operatorname{Hess}(r)\left(e_{i}, e_{i}\right)
\end{aligned}
$$

where $\operatorname{Hess}(r)$ is the Hessian of $r$. By the Gauss lemma, $\nabla r=\partial$,

$$
\nabla r^{-(a+b)}=-(a+b) r^{-(a+b+1)} \partial
$$

Substituting (4.4)-(4.6) into (4.3), one has

$$
2 I I=-\int_{M} \frac{|u|^{2}}{r^{a+b+1}}\left[\sum_{i=2}^{n} r \operatorname{Hess}(r)\left(e_{i}, e_{i}\right)-(a+b)\right] d v
$$

Let

$$
A=\int_{M} \frac{|u|^{2}}{r^{2 a}} d v \quad, \quad B=-2 I I \quad, C=\int_{M} \frac{|\nabla u|^{2}}{r^{2 b}} d v
$$

then (4.2) takes the form

$$
A t^{2}-B t+C \geq 0
$$

for every $t \in \mathbb{R}$ which implies that $B^{2}-4 A C \leq 0$. In other words,

$$
\frac{1}{2}\left|\int_{M} \frac{|u|^{2}}{r^{a+b+1}}\left[r \sum_{i=2}^{n} \operatorname{Hess}(r)\left(e_{i}, e_{i}\right)-(a+b)\right] d v\right| \leq\left(\int_{M} \frac{|u|^{2}}{r^{2 a}} d v\right)^{\frac{1}{2}}\left(\int_{M} \frac{|\nabla u|^{2}}{r^{2 b}} d v\right)^{\frac{1}{2}}
$$

This implies (1.8), since $\operatorname{Hess}(r)\left(e_{1}, e_{1}\right)=0$ and the trace of Hessian of $r$ is the Laplacian $\Delta r$ of $r$.

Since the radial curvature $K \leq 0$, it follows from the Hessian comparison theorem [7] or Theorem 5 that

$$
\sum_{i=2}^{n} r \operatorname{Hess}(r)\left(e_{i}, e_{i}\right) \geq n-1
$$


Hence via the assumption $a+b+1 \leq n$,

$$
\frac{1}{2} \int_{M} \frac{|u|^{2}}{r^{a+b+1}}(n-(a+b+1)) d v \leq\left(\int_{M} \frac{|u|^{2}}{r^{2 a}} d v\right)^{\frac{1}{2}}\left(\int_{M} \frac{|\nabla u|^{2}}{r^{2 b}} d v\right)^{\frac{1}{2}}
$$

This proves Theorem 3 .

To prove Theorem 4, we use the Hessian comparison theorem, and obtain

$$
\sum_{i=2}^{n} r \operatorname{Hess}(r)\left(e_{i}, e_{i}\right) \leq n-1
$$

By the assumption $a+b+1 \geq n$, Theorem 4 follows from (4.8).

\section{Embedding Theorems for weighted Sobolev Spaces of functions on Riemannian manifolds}

Following Costa's notation [6], we let $D_{\gamma}^{1,2}(M)$ denote the completion of $C_{0}^{\infty}\left(M \backslash\left\{x_{0}\right\}\right)$ with respect to the norm

$$
\|u\|_{D_{\gamma}^{1,2}(M)}:=\left(\int_{M} \frac{|\nabla u|^{2}}{r^{2 \gamma}} d v\right)^{\frac{1}{2}}
$$

$L_{\gamma}^{2}(M)$ denote the completion of $C_{0}^{\infty}\left(M \backslash\left\{x_{0}\right\}\right)$ with respect to the norm

$$
\|u\|_{L_{\gamma}^{2}(M)}:=\left(\int_{M} \frac{|u|^{2}}{r^{2 \gamma}} d v\right)^{\frac{1}{2}}
$$

and $H_{a, b}^{1}(M)$ denote the completion of $C_{0}^{\infty}\left(M \backslash\left\{x_{0}\right\}\right)$ with respect to the Sobolev norm

$$
\|u\|_{H_{a, b}^{1}(M)}:=\left(\int_{M}\left[\frac{|u|^{2}}{r^{2 a}}+\frac{|\nabla u|^{2}}{r^{2 b}}\right] d v\right)^{\frac{1}{2}}
$$

Theorem 6. Let $M$ be a manifold of radial curvature $K \leq 0$, or as in Corollary 1.6 with $a+b+1<n$, or as in Theorem 4, Corollary 1.7, or Corollary 1.8 with $a+b+1>n$, or as in Corollary 1.9 with $a+b+1 \neq n$. Then the following continuous embeddings hold

$$
H_{a, b}^{1}(M) \subset L_{\frac{a+b+1}{2}}^{2}(M) \quad \text { and } \quad H_{b, a}^{1}(M) \subset L_{\frac{a+b+1}{2}}^{2}(M)
$$




\section{Generalized inequalities on manifolds}

\section{Corollary 6.1.}

(i) For any $u \in H_{b+1, b}^{1}(M)$ it follows that

$$
\left(\frac{n}{2}-(b+1)\right)^{2} \int_{M} \frac{|u|^{2}}{r^{2(b+1)}} d v \leq \int_{M} \frac{|\nabla u|^{2}}{r^{2 b}} d v ;
$$

(ii) For any $u \in H_{a, a+1}^{1}(M)$ it follows that

$$
\left(\frac{n}{2}-(a+1)\right)^{2} \int_{M} \frac{|u|^{2}}{r^{2(a+1)}} d v \leq\left(\int_{M} \frac{|u|^{2}}{r^{2 a}} d v\right)^{\frac{1}{2}}\left(\int_{M} \frac{|\nabla u|^{2}}{r^{2(a+1)}} d v\right)^{\frac{1}{2}}
$$

(iii) If $u \in H_{-(b+1), b}^{1}(M)$ then $u \in L^{2}(M)$ and

$$
\left(\frac{n}{2}\right) \int_{M}|u|^{2} d v \leq\left(\int_{M} r^{2(b+1)}|u|^{2} d v\right)^{\frac{1}{2}}\left(\int_{M} \frac{|\nabla u|^{2}}{r^{2 b}} d v\right)^{\frac{1}{2}}
$$

(iv) If $u \in H_{0,1}^{1}(M)$, then $u \in L_{1}^{2}(M)$ and

$$
\frac{n-2}{2} \int_{M} \frac{|u|^{2}}{r^{2}} d v \leq\left(\int_{M}|u|^{2} d v\right)^{\frac{1}{2}}\left(\int_{M} \frac{|\nabla u|^{2}}{r^{2}} d v\right)^{\frac{1}{2}}
$$

(v) If $u \in H_{-1,1}^{1}(M)$, then $u \in L_{\frac{1}{2}}^{2}(M)$ and

$$
\left(\frac{n-1}{2}\right) \int_{M} \frac{|u|^{2}}{r} d v \leq\left(\int_{M} r^{2}|u|^{2} d v\right)^{\frac{1}{2}}\left(\int_{M} \frac{|\nabla u|^{2}}{r^{2}} d v\right)^{\frac{1}{2}}
$$

(vi) If $u \in H^{1}(M)=H_{0,0}^{1}(M)$, then $u \in L_{\frac{1}{2}}^{2}(M)$ and

$$
\left(\frac{n-1}{2}\right) \int_{M} \frac{|u|^{2}}{r} d v \leq\left(\int_{M}|u|^{2} d v\right)^{\frac{1}{2}}\left(\int_{M}|\nabla u|^{2} d v\right)^{\frac{1}{2}}
$$

Remark 6.1 The case $M=\mathbb{R}^{n}$ is due to [6].

Proof. We make special choices in (1.9) or (1.10) as follows:

(i) Let $a=b+1$;

(ii) Let $b=a+1$;

(iii) Let $a=-b-1$;

(iv) Let $a=0, b=1$;

(v) Let $a=-1, b=1$;

(vi) Let $a=0, b=0$. 


\section{Topological applications}

Using the same idea as in Proposition 5.1 in [17], one obtains

Theorem 7. Let $M$ be an $n$-manifold. If $M$ supports inequality (1.4) with $n>p$ (resp. (1.6) with $n<p$ ) for every $u \in W_{0}^{1, p}(M)$, then $M$ is not compact.

Proof. If $M$ were compact, then substituting $u \equiv 1$ into (1.4) (resp. (1.6)) we would have $\int_{M} \frac{|u|^{p}}{r^{p}} d v=0$, or $u=0$ a.e. This is a contradiction.

\section{Geometric applications}

Since geometric inequalities are linked to topology, and since curvature is related to topology, we have the following geometric application:

Theorem 8. Let $M$ be a complete manifold (Let $M$ be an $n$-manifold with $n>p$, and $x_{0} \in M$. If $M$ supports inequality (1.4)(resp. (1.6) with $\left.n<p\right)$ for every $u \in W_{0}^{1, p}(M)$, then there does not exists a constant $\tau>0$ such that the Ricci curvature $\operatorname{Ric}^{M} \geq \tau$.

Proof. Suppose on the contrary, then by Bonnet-Myers' Theorem (cf. $[3,16]), M$ would be compact. This contradicts Theorem 7 .

\section{References}

[1] W. Ambrose, Parallel translation of Riemannian curvature, Ann. Math. 64 (1956), 337-363.

[2] S. Bochner, Vector fields and Ricci curvature, Bull. Amer. Math. Soc. 52 (1946), 776-797.

[3] O. Bonnet, Sur quelques propriétés des lignes géodésiques, C.R. Ac. Sc. Paris 40(1855), 1311-1313.

[4] E. Cartan, Géométrie des espaces de Riemann, Gauthier-Villars, Paris, 1946.

[5] L. Caffarelli, R. Kohn and L. Nirenberg, First order interpolation inequalities with weights, Compos. Math. 53 (1984), 259-275.

[6] D. G. Costa, Some new and short proofs for a class of Caffarelli-Kohn-Nirenberg type inequalities, J. Math. Anal. Appl. 337 (2008), 311-317.

[7] R. E. Greene and H. Wu, Function theory on manifolds which posses a pole, Lecture Notes in Math. 699 (1979), Springer-Verlag.

[8] G. H. Hardy, J. E. Littlewood, G. Polya, Inequalities, Cambridge Univ. Press, Cambridge, UK, 1952.

[9] N. Hicks, A Theorem on Affine Connections, Illinois J. Math. 3 (1959), 242-254.

[10] N. Hicks, Connexion preserving spray maps, Illinois J. Math. 10 (1966), 661-679.

[11] L. Hörmander, An Introduction to Complex Analysis in Several Complex Variables, Van Nostrand, 1966

[12] R. Howard and S. W. Wei, Inequalities relating sectional curvatures of a submanifold to the size of its second fundamental form and applications to pinching theorems for submanifolds, Proc. Amer. Math. Soc. 94 (1985), 699-702. 
[13] K. Kodaira, On a Differential-Geometric method in the theory of analytic stacks, Proc. Nat. Acad. Sci. U.S.A. 39 (1953), 1268-1273.

[14] C. S. Lin, Interpolation inequalities with weighted norms, Comm. Partial Differential Equations 11 (1986), 1515-1538.

[15] E. Mitidieri, A simple approach to Hardy inequalities, Mathematical Notes 67 (2000), 479486.

[16] S. B. Myers, Riemannian manifolds with positive mean curvature, Duke Math. J. 8 (1941), 401-404.

[17] S. W. Wei, Nonlinear partial differential systems on Riemannian manifolds with their geometric applications, Journal of Geometric Analysis 12 (2002), 147-182

Department of Mathematics, University of Oklahoma, Norman, Oklahoma 73019-0315, U.S.A.

E-mail: wwei@ou.edu

Department of Mathematics, University of Oklahoma, Norman, Oklahoma 73019-0315, U.S.A.

E-mail: yli@math.ou.edu 